\title{
Infrared sound and music controller for users with specific needs
}

\author{
B P Challis ${ }^{1}$ and $\mathrm{K}^{\text {Challis }}{ }^{2}$ \\ ${ }^{1}$ School of Creative and Cultural Industries, University of Glamorgan, \\ Adam Street, Cardiff, UK \\ ${ }^{2}$ Learning Support Service, Education Bradford, \\ Future House, Bradford, UK \\ bchallis@glam.ac.uk,kate.challis@educationbradford.com
}

\begin{abstract}
The design and rationale behind a novel music and sound controller ("The Benemin") is described. Using an array of eight low-cost infrared distance measuring sensors, the system enables users to trigger and manipulate sounds using MIDI messages. Although the controller can facilitate complex musical interaction, providing eight note polyphony and expressive control, the central theme of the project has been one of accessibility. The controller is designed to be used in a variety of settings by users with special needs and has been designed to be both intuitive to play and easy to set up. An ongoing programme of user testing is described and discussed alongside preliminary results.
\end{abstract}

\section{INTRODUCTION}

There are two key hardware systems in regular use within the UK that are aimed at bridging the gap between an individual's desire to interact with sound and the physical and cognitive barriers that can obstruct that same desire. The first, and probably the most commonly available technology, is Soundbeam. This uses an ultrasonic sensor to create an invisible 'beam' that is mapped to musical pitches along its length. The other system, MIDI Creator, allows various types of sensor to be utilised for the same purpose; this includes an ultrasound sensor similar to that used by Soundbeam. Both systems provide immediate interest by allowing the user to produce and interact with sounds that are triggered by MIDI messages. With this in mind, both systems can be regarded as inspirational and accessible music systems where the kinds of interaction encouraged are achievable by individuals with limited mobility. Additionally, when working with individuals with severe learning difficulties, such devices can be a particularly intuitive method for exploring and encouraging cause-and-effect style interaction. At a higher level, both systems can also be thought of as musical instruments where the notes produced are being manipulated to create melodic fragments and rhythms. Again, the type of interaction afforded is flexible to the needs of users with limited mobility. However, when systems such as these are considered as musical instruments in this way it becomes important to consider their relative merits within a context of structured music-making activities. It is not the intention of this paper to unduly criticise either of these two systems, indeed it is acknowledged that both offer strong music making potential. However, comments and observations gathered from practitioners working within special needs education suggest that there would be a place for a more dedicated musical instrument of this nature that offers similar levels of accessibility but with less complexity in terms of setup and operation.

\subsection{Musical Tasks}

At the most basic of levels, the building blocks of western music can be regarded as pitch, harmony and rhythm. However, the combination of pitch and rhythm provides us with melody and the layering of pitches provides us with harmony and it is typically the combination of these two along with rhythm that are used when making specific reference to musical ideas. However, the types of musical instruments that we use and the way in which we perform with them also provides us with expressive qualities such as timbre and loudness. Indeed, the level of expression afforded by one instrument in comparison to another can be just as significant as the different types of musical task that either can achieve. In terms of accessibility and music, it would be desirable for any enabling-instrument to be as flexible as possible in terms of the different types of musical task that can be achieved whilst also offering considerable expressive control. 
One quite fundamental musical task would be to copy or improvise a rhythmic pattern. For this task, the sound used does not need to be pitched but the player will need to be able to move freely from one sound to another at specific times. With Soundbeam, this can be limiting as to move from one sound to another the user must either move within the beam (triggering any sounds between the ones being targeted) or remove the hand or other body part being used and re-enter the beam at an alternate location; this can be difficult to gauge. MIDI creator has the same limitations when using the ultrasound sensor but is less dedicated in its design such that there is clear potential to use a number of sensors of different types for different purposes. However, this requires the user to decide which sensors will be used and how they will function; this creates additional levels of complexity in terms of ease-of-use. It also requires that a variety of different sensors be readily available.

If a rhythmic task is performed but the sounds used have pitch, then the task becomes melodic. Melody will typically be associated with tonality and a musical key. Both Soundbeam and MIDI Creator allow predefined or programmable scales to be mapped across the sensor(s). Although, the earlier limitation remains true Soundbeam, in particular, is effective at creating musical textures where it may not be of significance whether the musical ideas are, in part, produced as a result of a rule-based performance behaviour. There are numerous inbuilt functions within the system for creating melodic, rhythmic and harmonic ideas but access to these depends on how adept an individual is at understanding and programming the system.

Finally, if more than one pitch is played at the same time the task can be thought of as harmonic. An instrument's ability to produce harmonic sounds is limited by its polyphony. For example, a flute is monophonic, producing only one note at a time whereas a standard guitar is six note polyphonic allowing chords to be sounded. In this respect, Soundbeam is essentially monophonic although it is possible to sound chords that have been programmed into the system. MIDI Creator has the potential to be polyphonic but again requires additional levels of customisation to make this possible.

A basic requirement for an instrument to be expressive would be that individual notes can be sounded at different levels of loudness. On some acoustic instruments, the loudness of a sustained note can be changed over time which is a particularly expressive quality. Additionally, it is often possible to alter the timbre of a note by adjusting the way in which it is played, again, this is a very expressive quality and one that can also be regarded as desirable.

\section{PROJECT AIMS}

\subsection{Overview}

The central aim of the "Benemin" project has been the design and realisation of a controller-style musical instrument that can afford complex levels of expression and interaction whilst also fulfilling the inspirational role that can be instrumental in attracting and maintaining a user's interest. At its most basic level it should operate as an 'inspirational' musical toy, an object that encourages users to interact because of the connection between the actions they are making and the sounds being produced (cause-and-effect). As identified earlier, this would have direct application within an educational setting for users with severe learning needs. At a higher level, it should be capable of more structured musical interactions such that users can shape and develop simple but coherent musical ideas (melodies, rhythms and simple harmonies). At a higher level still, it should operate as a performance tool that can facilitate more complex levels of musical and expressive control. In addition, the controller should be as accessible as possible, being flexible and adaptable enough to respond to different levels of mobility and dexterity on the part of the user. It should also be intuitive to use and simple to operate. Finally, it was also recognised that the overall cost of the instrument should be regarded as significant within the design process.

There would be advantages to be gained from such a system. Practitioners working in educational and/or community-based settings would have the potential to use a single instrument with users across a broad range of physical and cognitive abilities. This is also advantageous to education providers where specialist technology can often be very expensive; the more flexible the system is, the more likely that it can be used in a variety of settings. If the system is intuitive to use and simple to set up, it is likely that more staff will be able to easily use the equipment with an increased potential for autonomy on the part of the learner within educational activities. Key issues explored within the design process have been musicality, usability, accessibility and affordability. 


\subsection{Musicality}

As was discussed earlier, the more polyphonic an instrument is the more capable it will be in terms of producing rich and potentially complex harmonies. However, even being able to sound two notes simultaneously provides the basis for introducing a simple major or minor interval; this alone, can be enough to reinforce the tonality of a musical idea. With this in mind, it was identified that the instrument should be two-note polyphonic at minimum but with a view to increasing this figure if possible.

At minimum, it would be desirable to have control over the loudness of the notes being played with the instrument. However, many musical instruments allow dynamic control within the life of a single note (wind instruments in particular). For example, a saxophonist can sound a note and then control the loudness with increased or decreased airflow; this can also change the timbre of the sound being produced. This is a particularly expressive musical effect and yet one that is simple and intuitive to understand. It was decided that at minimum, the instrument should be able to achieve different levels of volume but that the possibility for dynamic change in the quality of the sound should also be explored.

\subsection{Usability and Accessibility}

The two devices described earlier (Soundbeam and MIDI Creator) both carry significant learning-curves in terms of acquiring an adequate understanding of how to operate, adapt and expand the system. This is an observation that has been offered by educators prior to the design process and has also been echoed further during the testing process. The feeling appears to be that these systems are not used as frequently as they might be as there may only be one member of staff within a school or institute who is fully knowledgeable in its use. However, the gesture-based approach to triggering sounds offered by these devices appears to be both intuitive and flexible in terms of facilitating access for users with limited mobility. Traditional musical instruments tend to require high levels of dexterity in terms of control and movement of individual fingers; they often require the use of both hands in this way. With this in mind, it was decided that a gesture-based approach to interaction where one or more hands (wrists, arms etc) could be used to play individual notes would be highly desirable but that the general complexity of the system should be kept as simple as possible.

\subsection{Affordability}

Specialist technology within the educational setting is often expensive and this factor alone can impact on whether individuals are exposed to a particular experience or not. It was decided that one of the aims of the project should be to try and keep the overall cost of the instrument as low as possible. One key aspect that was identified as being significant within this was the choice of technology to be used for sensing movement. Ultrasonic sensors can have considerable range and accuracy, however, they are also particularly costly unless considered within the context of mass-production. In contrast to using this type of technology, it was decided that the project should explore possibilities for the use of low-cost infrared sensors.

\section{DESIGN}

\subsection{Performance Behaviour}

According to Malloch et al. (2006), Digital Music Instruments (DMIs) can be categorised within three distinct modes of performance behaviours: skill-based, rule-based and model-based. Of these, the mode of musical interaction-behaviour most similar to that of playing a conventional musical instrument lies within the skill-based domain. The implication is that the user will be interacting in real-time in response to a continuous audio-stream. The other two models of musical interaction-behaviour operate at increasingly abstract levels of interaction with the user's interactions being less and less involved in terms of immediacy of output. Based on these definitions for performance behaviour, the central design consideration was for the system to employ a completely skill-based approach to interaction; a user's movements should be directly interacting with and affecting the sound that is output. Although it may be desirable to be able to create unusual and perhaps quite complex musical textures through the use of rule-based behaviours, this can be achieved separately with third-party software. With this in mind, it was decided at an early stage that the Benemin should be thought of simply as a diatonic instrument that is both accessible and expressive.

\subsection{Hardware}

The current working prototype is based on the Sharp GP2Dxx series of infrared (IR) distance measuring sensors (see Fig 1.). These are particularly versatile sensors that are commonly used in robotics for detecting objects within a limited field or 'beam'. Discussion on the relative merits of IR sensors is provided by 
O'Sullivan and Igoe (2004) and also by Miranda and Wanderley (2006). Essentially, each sensor unit includes an IR transmitter-receiver pair that provides continuous feedback on whether an object is within the 'beam' of the sensor. Although these have a shorter range than ultrasonic sensors they are less costly and also more self-contained. GP2Dxx sensors are available as digital output devices (providing logic-high or logiclow according to a distance threshold) or as analogue output devices (providing a voltage that represents the distance from the object to the sensor). These units are low-cost and require minimal additional electronics making it relatively easy to incorporate a number of them into an array. The unit in use within the current system provides analogue output for distances in the range $10 \mathrm{~cm}$ to $80 \mathrm{~cm}$.

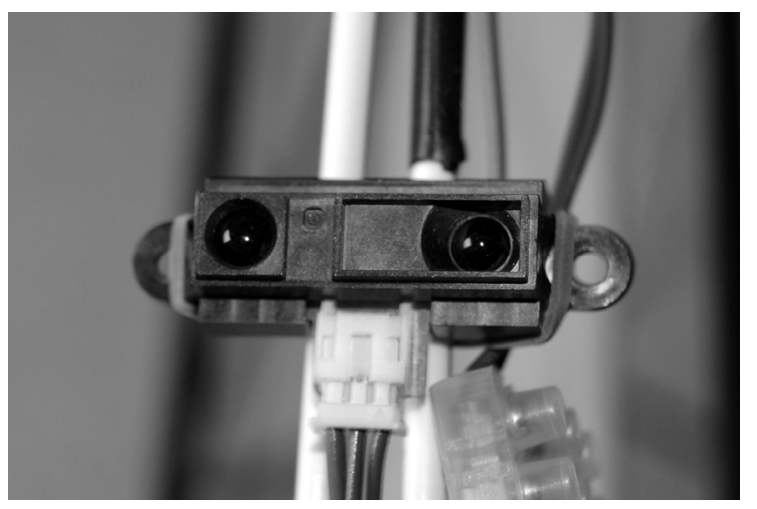

Figure 1. Sharp GP2Dxx series IR distance measuring sensor.

For the initial system, eight sensors have been attached in line to a light-weight curved-frame with the sensor 'beams' orientated upwards in front of the performer's body (see Fig. 2). This curved layout is for practical reasons rather than aesthetic as IR sensors of this type can have a tendency to trigger neighbouring units that are in close proximity if their beams overlap. The curvature of the frame aids in pointing the sensors away from each other slightly such that the distance between any two can be kept to a useable size (approximate to a hand's width). The overall height of the frame above its base is $22 \mathrm{~cm}$ and the overall span between the extreme sensors is $92 \mathrm{~cm}$ with a distance of approximately $14 \mathrm{~cm}$ between neighbouring sensors.

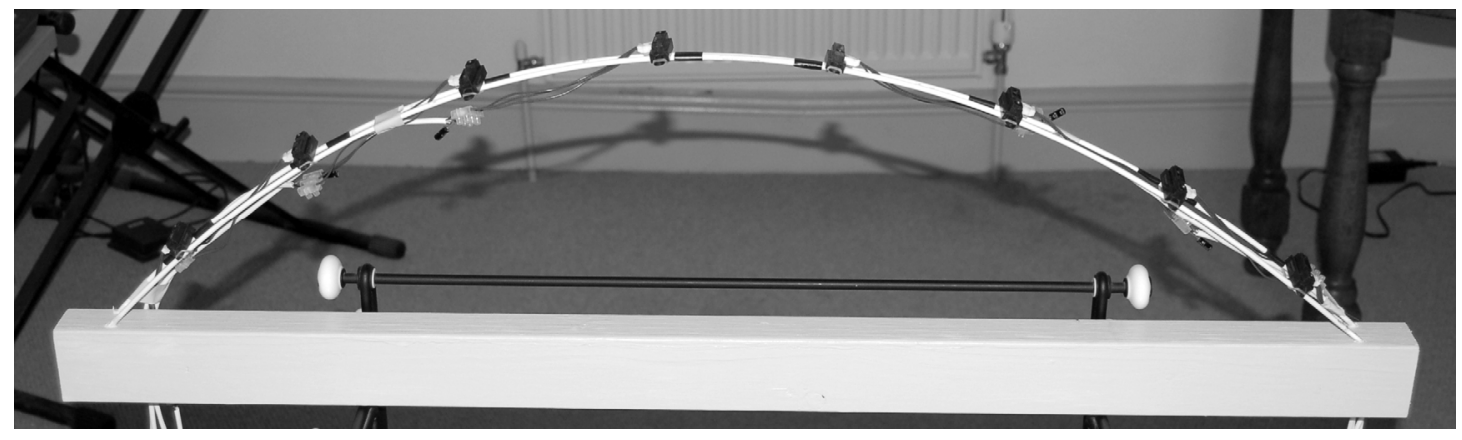

Figure 2. The Benemin.

A programmable microchip is used to run software that maps the change in voltage onto standard MIDI note and/or controller messages which are then relayed to any hardware or software sound-generating device that is connected. The system can be regarded as being eight note polyphonic as all sensors are read and acted upon independently of each other. However the actual polyphony achieved will, in part, depend on the capabilities of the hardware or software being controlled.

\section{$3.3 \quad$ Functionality}

The Benemin can be used in two different ways depending on the nature of the hardware it is controlling. In its first mode (instrument), the system simply transmits Note On and Off messages accordingly as an object enters or leaves a beam, these have a velocity (loudness) that corresponds to the distance from the sensor to the object (finger, hand, arm etc.). This is useful for working with standard MIDI sound modules or synthesisers with the controller functioning in a similar fashion to a MIDI keyboard. The pitches produced are currently taken from pre-defined scales and modes that are mapped from left to right (lowest pitch to highest pitch). In this mode, the controller can be thought of as a diatonic instrument where each note is a 
specific step within one of a number of available scales (major, natural minor, harmonic minor, pentatonic, blues, dorian, mixolydian etc.). Although, these scales are currently predefined, it would not be difficult to introduce some level of user programming to this aspect. In the current version, instrument is the default mode along with a scale mapping of $\mathrm{C}$ major starting at middle $\mathrm{C}$.

In its second mode (controller), the Note On and Off messages are still transmitted but there is an additional stream of controller messages available whilst the object remains within the beam. These change accordingly as the object is moved closer or further away from the sensor. The MIDI specification does not allow for individual note volumes to be changed dynamically but it is possible to incorporate this effect using sound programming environments such as MAX MSP and Reaktor (the full MIDI specifcation is available as a standalone resources but is covered in considerable depth in various general texts e.g. Huber (1998).) This mode provides the basis for achieving some level of expressive control. A Note On message can be used to trigger a sampled pitch or sound, the subsequent controller messages can then be used to alter the sound dynamically. This could be used to alter loudness of an individual note but could equally be used to alter its tone.

\section{TESTING}

\subsection{Overview}

As described earlier, the central aims of the project have been to design an accessible music controller, one that is easy to play and set up but also one that can accommodate a fairly broad range of user abilities and musical applications. This means that there are a number of different target groups that need to be considered within a context of user-testing. For the purposes of the testing programme for the Benemin, three contrasting user-groups have been identified. The first group includes users who have severe learning needs and in this context the Benemin is being seen as an inspirational device where simple cause-and-effect interaction can encourage a user to exercise and develop cognitive and motor skills. The second group includes users who have mild learning difficulties but probably also have some level of restricted mobility. The final group includes users where limited mobility is the most significant constraint.

A Specialist Teacher for pupils with multiple sensory impairment is working with a variety of users with differing individual needs using the Benemin system in its default operating mode (instrument as described earlier). Feedback is being gathered through close observation of individuals using the controller along with the critical evaluation of additional specialists working in the same environment. Group sizes are typically in the order of four or five individuals within a group but with generally only one person interacting with the device at any given time. For the purposes of the initial phase of testing there is no specific script being followed i.e. the testing is not task-specific. Where possible and appropriate, users are simply allowed to interact with and learn how to use the device in a way that works for them. It is important to appreciate that the system is currently being assessed within a working educational programme and, as such, intervention from the observer is acknowledged as appropriate. With this in mind, where an individual has perhaps struggled to comprehend or interact easily with the system it would be both acceptable and appropriate for the individual to be encouraged to attempt a different action. The difficulties that were apparent are then recorded. It is envisioned that the majority of the data gathered will be qualitative in nature.

\subsection{Preliminary Results}

At present, the testing programme is focusing on the first two user groups. A selection of individuals from the third user group have been identified to take part in testing. They have been working for some time with a specialist music therapist experiencing various musical activities using conventional instruments and technology. It is anticipated that this will be a particularly rich source of feedback but testing with this group will not be possible until later this year.

One group of young adults from the first user group has been observed working with the Benemin but observation within the group has quickly demonstrated that the current shape of the device is not as effective as was originally anticipated. This is not completely surprising as the curved shape, height, width and layout of sensors on the first prototype are based on relatively arbitrary measurements. There are a number of issues that are worth discussing here, the first being that the overall height of the sensor array can be quite awkward to reach for a user with severely limited movement. This is because the array has been mounted on a curved frame to point neighbouring sensors away from each other to reduce spurious triggering. The slope of the curvature can be reduced significantly to help make this less of an issue. In a similar way, the width of the array is also an issue in terms of reaching the extremities. The width, in part, is governed by the number of 
sensors in use. Eight sensors are available but really only to provide enough notes to produce usable musical scales. When used in a cause-and-effect style activity this number could be greatly reduced.

Some of the individuals from this group were in wheelchairs and it has been suggested that the curved shape of the frame might be an asset for this type of use if the base could be removed. Ultimately, it was also quite apparent that the body of the prototype is simply not robust enough to be used effectively with this particular user group yet and that this clearly needs to be addressed before testing can be continued adequately and safely. Although it is disappointing that the results with this group have not been as immediately promising as had been hoped they are greatly informative and are contributing significantly to the redesign of the overall layout and shape of the device.

In contrast, testing with two further groups made of individuals from the second user-group has produced very promising and encouraging results. These groups have included young adults with moderate learning difficulties but who are generally more mobile. Most noticeably, the issues outlined as a result of testing with the individuals from user-group one have not appeared to be as significant for those from user-group two. Individuals within these groups were allowed to simply try the instrument and improvise with the sounds as they wished. It has been rewarding to observe that users have been able to exhibit considerable independence whilst improvising with the system, although one key observation on this has been that it would be desirable to integrate a number of easily accessible switches that change one or two very basic settings e.g. type of sound and/or type of musical scale.

Users have also been observed trying to interact with the system in contrasting ways. For example, one user began by attempting a 'tapping' motion towards the sensors and then gradually adopted a more appropriate hand motion above the sensors. Another user, was using a gentle pushing movement towards the sensors which sometimes failed to trigger the sensor. Yet another, began at one end of the instrument with the sensors making a line perpendicular to their body, similar to the strings on a harp. Although the original design of the instrument had a particular style of gesture in mind it must now be observed that there are likely to be a number of alternate styles of interaction that might be more intuitive or, perhaps, physically less demanding depending on the abilities of the user. These are being observed and recorded during the closeobservation sessions such that they can be compared and contrasted with one another in future designs.

Some users have also been observed moving their hands towards or away from sensors whilst a note is currently sounding; this is an apparent attempt to alter the character of the sound in some way. As identified earlier, the ability to dynamically change the loudness or tone of a sustained note can be a simple yet very expressive device. Although this feature is available within the system it is not yet a default setting. It seems that if the feature were to be made available in this way that it would be both instinctive and intuitive in operation. By placing an object into a 'beam' a sound is produced, if the object is then moved then the tone or volume of sound is affected to some degree. This is an aspect that will be explored further during follow up design phases.

Testing is also being carried out within a series of ongoing live improvised performances by users without specific needs. The aim here is simply to monitor how well the system performs (e.g. sensitivity, usability, reliability, interference etc.) within the context of specific performance activities. This has been particularly useful in terms of identifying a number of software 'bugs' but also in terms of assessing the impact of different lighting conditions on the reliability of the sensors in use. It is anticipated that all of these results will be greatly enhanced by the testing that involves individuals from user-group three.

\section{FURTHER WORK}

There are obvious issues with the shape and the layout of the current Benemin that need addressing before the instrument can be adequately reassessed with individuals from user-group one. The first is to reduce the height of the curve in the frame and the second is to reduce the width of the instrument's span. Lowering the height will be relatively easy but this needs to be adjusted in relationship to the spacing of the sensors such that they do not interfere with one another. It is anticipated that this can probably be reduced significantly but there is also now a desire to maintain a level of curvature in the frame as this could be beneficial for users in wheelchairs. However, the base would need to be removed to maximise the flexibility that the curvature could provide in terms of positioning the instrument above a user's lap. It may be possible to redesign the frame so that it is supported by an adjustable stand such that it can be set to different heights for different users.

There appears to be no immediate need to either increase or decrease the number of sensors available within the array for user-group two. Eight sensors allows many familiar musical scales and modes to be 
explored and this appears to contribute to the general appeal of the interaction. However, with user-group one, it would appear that fewer sensors could be less confusing and this would also reduce the overall span of the instrument. There are a number of possible solutions that are to be explored here. One would be to somehow make the instrument modular such that different numbers of sensors can be physically positioned and connected to suit individual needs. An alternate method would be to keep the general layout as eight sensors but to allow for individual sensors to be turned on or off. At its most extreme, this could mean that perhaps only one or two sensors might be set to be active and it might be that these are ones that most suit the type and level of movement that the user is most comfortable with.

\section{CONCLUSION}

Initial testing of the Benemin system has shown that the device has considerable potential as an accessible musical instrument for users with specific needs. However, this has mainly been observed whilst the device has been in use by users with moderate learning difficulties and marginally limited mobility. There have been indications that expressive control over individual notes would be used instinctively if the interaction by which this is achieved is intuitive to the user. Where users with severe learning difficulties were involved the layout and general shape of the device has been shown to require further consideration. The most significant issues recorded were with the height and width of the device and also the general robustness of the build. These are all aspects that can be significantly enhanced within a revised design and this is currently being addressed. A third user-group containing individuals where limited mobility is the prime consideration are still to be assessed with the device in its current form.

\section{REFERENCES}

D M Huber (1998), The MIDI Manual, Second Edition, Focal Press.

E R Miranda and M M Wanderley (2006), New Digital Musical Instruments: Control And Interaction Beyond the Keyboard, A-R Editions.

J Malloch and D Birnbaum and E Sinyor and M Wanderley (2006), Towards a New Conceptual Framework for Digital Musical Instruments, Article in: Proceedings of the $9^{\text {th }}$ International Conference on Digital Audio Effects.

D O’Sullivan and T Igoe (2004), Physical Computing, Thompson Course Technology, Boston MA. 